\title{
Dark matter as the Bose-Einstein condensation in loop quantum cosmology
}

\author{
K. Atazadeh ${ }^{1, \mathrm{a}}$, F. Darabi ${ }^{1,2, \mathrm{~b}}$, M. Mousavi ${ }^{1, \mathrm{c}}$ \\ ${ }^{1}$ Department of Physics, Azarbaijan Shahid Madani University, Tabriz 53714-161, Iran \\ ${ }^{2}$ Research Institute for Astronomy and Astrophysics of Maragha (RIAAM), Maragha 55134-441, Iran
}

Received: 5 May 2016 / Accepted: 7 June 2016 / Published online: 15 June 2016

(C) The Author(s) 2016. This article is published with open access at Springerlink.com

\begin{abstract}
We consider the FLRW universe in a loop quantum cosmological model filled with radiation, baryonic matter (with negligible pressure), dark energy, and dark matter. The dark matter sector is supposed to be of Bose-Einstein condensate type. The Bose-Einstein condensation process in a cosmological context by supposing it as an approximate first-order phase transition, has already been studied in the literature. Here, we study the evolution of the physical quantities related to the early universe description such as the energy density, temperature, and scale factor of the universe, before, during, and after the condensation process. We also consider in detail the evolution era of the universe in a mixed normal-condensate dark matter phase. The behavior and time evolution of the condensate dark matter fraction is also analyzed.
\end{abstract}

\section{Introduction}

When one considers a universe following the standard Einstein cosmology, that is, when the dynamics of the universe is described by the general relativity equations, one deduces that there exists a mysterious singularity at the beginning of time, the so-called big bang. This singularity may be considered as a deficiency of Einstein cosmology at high energies [1]. Actually, one can assert that the big bang implies the breakdown of general relativity at scales with high energies, whereas we know from the observational evidence, such as the existence of the cosmic microwave background, that the big bang model works at scales lower than Planck energy scale. At these scales, the universe was full of a hot photonbaryon combination almost described by a radiation fluid. This hot combination was cooling down as the universe was

\footnotetext{
a e-mail: atazadeh@azaruniv.ac.ir

be-mail: f.darabi@azaruniv.edu

c e-mail: mousavi@azaruniv.ac.ir
}

experiencing the expansion, and due to the different scaling behaviors of pure radiation and pure matter, the energy density of non-relativistic matter started to dominate over the energy density of radiation and led to the formation of structures.

Loop quantum gravity (LQG) is one of the potential candidates for the study of quantum gravity [2]. Homogeneous and isotropic space time, reduces loop quantum gravity LQG to loop quantum cosmology (LQC) [3]. According to this theory, due to the effective quantum gravitational effects, the big bounce happens to remove the big bang singularity, after which a super-inflation phase occurs, and then the universe enters a normal inflation regime [4,5]. The loop quantum cosmology effects manifest themselves in a new effective form of the modified Friedmann equation. These effective equations have been derived in the literature for FLRW loop quantum cosmologies considering different matter sectors in the early universe [6-12].

An extended scenario of the matter bounce cosmology, considering a single scalar field with an approximately exponential potential, was proposed as an alternative to the slow roll inflation, in the context of LQC, in which the universe has experienced a quasi-matter contracting phase with a variable equation of state parameter [13]. This matter bounce scenario, in the teleparallel version of LQC, was shown to be in good agreement with the new BICEP2 data [14]. A Gauss-Bonnet extension of LQC, by introducing holonomy corrections in modified $f(G)$ theories of gravity was also developed, where the authors have provided a perturbative expansion in the critical density as well as a parameter characteristic of LQG, and one obtained leading-order corrections to the classical $f(G)$ theories of gravity. They also presented a reconstruction method which makes possible to find the LQC corrected $f(G)$ theory capable of realizing various cosmological scenarios [15]. In another work, in order to avoid singularities of $f(R)=R+a R^{2}$ model, holonomy corrections to this model were introduced in Einstein 
frame and a detailed analytical and numerical study was performed when holonomy corrections are taken into account in both Jordan and Einstein frames. They obtained, in Jordan frame, a dynamics which is different qualitatively, from the one of the original model, at early times. According to this dynamics, the universe is not singular, neither at early times in the contracting phase, nor at bouncing to enter in the new expanding inflationary phase. This dynamics may lead to better predictions for the inflationary phase in comparison to the current observations [16].

Motivated by the above mentioned success of LQC in describing the very early stage of inflationary universe, we are encouraged to study the evolution of the universe after the inflationary era and investigate the possible establishment of a specific kind of dark matter (see below) and study its contribution in the subsequent evolution of the universe. Explicitly, we assume that after inflation the universe includes ordinary dark matter (bosonic particles), dark energy, radiation, and baryonic matter, and that the ordinary dark matter component in the sufficiently cooled universe has gone through a phase transition provided by the Bose-Einstein condensation, which might have happened during the early stages of cosmological evolution of the universe with a low temperature comparable to the critical temperature for Bose-Einstein condensation $T_{\mathrm{cr}} \sim 2 \pi \hbar^{2} n^{2 / 3} / m k_{\mathrm{B}}$ where $m$ is the particle mass, $n$ is the particle density and $k_{\mathrm{B}}$ is Boltzmann's constant [17-32].

Particularly, in the Bose-Einstein Condensation (BEC) model, the dark matter can be described as a non-relativistic Newtonian gravitational condensate, whose pressure and energy density are related by a barotropic equation of state. Based on the above arguments, we assume the possibility that the Bose-Einstein condensation might have happened during the early stages of cosmological evolution of the universe with a temperature comparable to the critical temperature for Bose-Einstein condensation $T_{\mathrm{cr}} \sim 2 \pi \hbar^{2} n^{2 / 3} / m k_{\mathrm{B}}$ where $m$ is the particle mass, $n$ is the particle density, and $k_{\mathrm{B}}$ is Boltzmann's constant [17-32].

The modification of cosmological evolution of the early universe, in the presence of condensate dark matter in standard cosmology, has already been studied in [33]. In this paper, we generalize this model from standard cosmology to LQC. The organization of the present paper is as follows. In Sect. 2, we outline the modification of Friedmann equations in LQC model and the basic properties of the normal and BEC dark matter in addition to the presentation of the relevant physical quantities and the equation of state. In Sect. 3 , we give a brief explanation about the cosmological dynamics of the Bose-Einstein condensate. Next, we introduce the numerical values of the cosmological parameters at the condensation point, and then obtain the important $h(t)$ parameter as a volume fraction of matter in the Bose-Einstein condensed phase which explains the evolution of the dark matter energy density during the transition process, and extract the order of BEC time interval in LQC model. In the following, we study the post condensation phase in LQC model describing the evolution of the scale factor parameter under the effect of the existence of the BEC dark matter in LQC. The paper ends with a conclusion.

\section{Bose-Einstein condensate dark matter in the LQC universe}

In this section, we intend to review the Bose-Einstein condensation and explore the dynamics of the condensation and some of the relevant cosmological implications. We consider the line element of the flat Friedmann-Robertson-Walker metric

$\mathrm{d} s^{2}=-c^{2} \mathrm{~d} t^{2}+a^{2}(t)\left(\mathrm{d} x^{2}+\mathrm{d} y^{2}+\mathrm{d} z^{2}\right)$,

where $a$ is the scale factor presenting the cosmological expansion. We describe the matter by the perfect fluid energymomentum tensor,

$T^{\mu \nu}=(\rho+p) u^{\mu} u^{\nu}+p g^{\mu \nu}$.

where $(\rho, p)$ stands for $\left(\rho_{b}, p_{b}=0\right),\left(\rho_{\text {rad }}, p_{\text {rad }}\right)$, and $\left(\rho_{\chi}\right.$, $\left.p_{\chi}\right)$ for baryonic matter, radiation and dark matter, respectively. Note that we do not consider the interaction between these energy terms, in other words the energy of these components are individually satisfying the conservation equation.

Taking into account the well-known loop quantum gravity constraints and coupling the matter to classical phase space of FLRW universe, we obtain [34-39]

$\dot{V}=\frac{3 V}{\lambda \gamma} \sin (\lambda b) \cos (\lambda b)$,

$\mathcal{H}_{\text {matt }}=\frac{3}{4 \gamma \lambda^{2}} V \sin ^{2}(\lambda b)$,

where $\mathcal{H}_{\text {matt }}$ is the matter Hamiltonian, $\gamma \approx 0.24[40,41]$ represents the Barbero-Immirzi parameter, and $\lambda \approx 2.27$ is the length gap. Meanwhile, $b$ is the conjugate momentum and $V$ is proportional to the physical volume of a cubical cell, with unit comoving volume $V=\frac{a^{3}}{2 \pi \gamma}$. A dot means the derivative with respect to the time. By rewriting Eqs. (3) and (4), we have

$$
\begin{aligned}
& H=\frac{\sin (2 \lambda b)}{2 \gamma \lambda}, \\
& \frac{\sin ^{2}(\lambda b)}{\gamma^{2} \lambda^{2}}=\frac{8 \pi}{3} \rho,
\end{aligned}
$$

where $H$ is the Hubble parameter and the matter density is limited as $\rho<\rho_{c}$ where $\rho_{c}=\frac{3}{8 \pi \gamma^{2} \lambda^{2}}$. Combination of (5) and (6) reduces to the modified FLRW equation in the loop quantum cosmology model [42-46] 
$3 \frac{\dot{a}^{2}}{a^{2}}=8 \pi G \rho\left(1-\frac{\rho}{\rho_{c}}\right)$.

As we mentioned, the effective equations have been derived for FLRW loop quantum cosmologies considering different matter sectors in the early universe [6-12]. So, we may use the modified FLRW equation in the loop quantum cosmology model, namely Eq. (7), and assume the matter density $\rho$ as the combination of different matter components $\rho_{b}, \rho_{\mathrm{rad}}, \rho_{\chi}$, and $\Lambda$ indicating the baryonic matter, radiation, dark matter, and cosmological constant, respectively, so that

$$
\begin{aligned}
3 \frac{\dot{a}^{2}}{a^{2}}= & 8 \pi G\left(\rho_{b}+\rho_{\mathrm{rad}}+\rho_{\chi}+\Lambda\right) \\
& \times\left(1-\frac{\left(\rho_{b}+\rho_{\mathrm{rad}}+\rho_{\chi}+\Lambda\right)}{\rho_{c}}\right) .
\end{aligned}
$$

The second modified FLRW equation is also given by

$$
\begin{aligned}
2 \frac{\ddot{a}}{a}+ & \frac{\dot{a}^{2}}{a^{2}}=8 \pi G \\
& \times\left(\frac{\left(\rho_{b}+\rho_{\mathrm{rad}}+\rho_{\chi}\right)^{2}-\Lambda^{2}}{\rho_{c}}+\Lambda-\frac{\left(p_{\mathrm{b}}+p_{\mathrm{rad}}+p_{\Lambda}\right)}{c^{2}}\right. \\
& \left.\times\left(1-2 \frac{\left(\rho_{b}+\rho_{\mathrm{rad}}+\rho_{\chi}+\Lambda\right)}{\rho_{c}}\right)\right)
\end{aligned}
$$

and also for the energy density conservation equation we have

$\dot{\rho}_{i}+3\left(\rho_{i}+\frac{p_{i}}{c^{2}}\right) \frac{\dot{a}}{a}=0, \quad(i \equiv b, \operatorname{rad}, \chi)$.

Now, regarding the cosmological evolution of radiation and baryonic matter we consider the relations $\rho_{\text {rad }}=$ $\rho_{\text {rad }, 0} /\left(a / a_{0}\right)^{4}$ and $\rho_{b}=\rho_{b, 0} /\left(a / a_{0}\right)^{3}$, respectively; meanwhile $\rho_{b, 0}$ and $\rho_{\mathrm{rad}, 0}$ are the energy densities corresponding to their values at $a=a_{0}$, and also for the dark matter we work with a general form of density as $\rho_{\chi}=\rho_{\chi}, 0 / f\left(a / a_{0}\right)$. It should be noted that $f\left(a / a_{0}\right)$ is an arbitrary function which depends on the special dark matter model. As we know we have the critical density $\rho_{\mathrm{cr}, 0}=3 H_{0}^{2}$, where $H_{0}$ is the value of the Hubble parameter at $a=a_{0}$. In addition, we can write the dimensionless parameters known as density parameters as $\Omega_{i, 0}=\rho_{i, 0} / \rho_{\mathrm{cr}, 0}$, with $i=b, \mathrm{rad}, \chi$. Using these relations helps us to write the following new form of the modified FLRW equation:

$$
\begin{aligned}
\left(\frac{\dot{a}}{a}\right)^{2}= & H_{0}^{2}\left(\frac{\Omega_{b, 0}}{\left(\frac{a}{a_{0}}\right)^{3}}+\frac{\Omega_{\mathrm{rad}, 0}}{\left(\frac{a}{a_{0}}\right)^{4}}+\frac{\rho_{\chi, 0}}{f\left(\frac{a}{a_{0}}\right)}+\Omega_{\Lambda}\right) \\
& -\frac{H_{0}^{4}}{\rho_{c}}\left(\frac{\Omega_{b, 0}}{\left(\frac{a}{a_{0}}\right)^{3}}+\frac{\Omega_{\mathrm{rad}, 0}}{\left(\frac{a}{a_{0}}\right)^{4}}+\frac{\rho_{\chi, 0}}{f\left(\frac{a}{a_{0}}\right)}+\Omega_{\Lambda}\right)^{2},
\end{aligned}
$$

where $\Omega_{\Lambda}$ is the dark energy density parameter. Thus, we can write the constraint

$\Omega_{b, 0}+\Omega_{\mathrm{rad}, 0}+\Omega_{\chi, 0}+\Omega_{\Lambda}=1$.
Now, we suppose that in the early universe the bosonic particles, with mass $m_{\chi}$ and a very high temperature $T$, were in equilibrium state with other particles in a hot relativistic plasma. After the expansion of the universe and rapid decrease of the temperature it was decoupled from this equilibrium state at a high chemical potential $\mu \gg m_{\chi}$ or low decoupling temperature $T_{D} \ll T$. At this decoupling temperature, we assume that the bosonic particles are still in kinetic equilibrium among themselves in an isotropic gas with low temperature. Consequently, for the spatial number density we have

$n=\frac{4 \pi g}{h^{3}} \int p^{2} f(p) \mathrm{d} p$,

where $g$ is the helicity state number, $h$ is Planck's constant, and $f(p)$ is defined as follows:

$f(p)=\frac{1}{(\exp [E-\mu]-1)}$,

where $E$ is the energy $E=\sqrt{p^{2}+m_{\chi}^{2} c^{4}}$ and $p$ is the momentum of the particle. Due to the redshift in the momentum of particles, we have $p(t) a(t)=p_{D} a_{D}$ where $p_{D}$ and $a_{D}$ denote for the momentum of particles and the scale factor of the universe, respectively, at the temperature $T_{D}$ denoting for the decoupling temperature from the rest of plasma. Besides, we have the scaling evolution relation for the number density given by $n_{\chi} \sim a^{-3}[47,48]$. For the extreme-relativistic case $E \approx p c\left(\mu=\mu_{D} a_{D} / a\right.$ and $\left.T=T_{D} a_{D} / a\right)$, the distribution function is derived as $f_{E R}(p)=(\exp [(p c-\mu)]-1)^{-1}$, hence for the non-relativistic case $E-\mu \approx p^{2} / 2 m_{\chi}-\mu_{k i n}$ $\left(\mu_{k i n} \equiv \mu-m_{\chi} c^{2}\right)$ the distribution function is given by $f_{\mathrm{NR}}(p)=\left(\exp \left[\left(p^{2} / 2 m_{\chi}-\mu_{k i n}\right)\right]-1\right)^{-1}$, where the evolution equations are $\mu_{k i n}=\mu_{k i n, D}\left(a_{D} / a\right)^{2}$ and $T=$ $T_{D} /\left(a_{D} / a\right)^{2}[47,48]$. For a frozen distribution of dark matter, the associated energy density $\epsilon$ and kinetic energymomentum tensor $T_{v}^{\mu}$ are as follows, respectively:

$\epsilon=\frac{g}{3 h^{3}} \int E f(p) \mathrm{d}^{3} p$,

$T_{v}^{\mu}=\frac{g}{h^{3}} \int \frac{p^{\mu} p_{v}}{p^{0}} f(p) \mathrm{d}^{3} p$.

The mentioned pressure is described by

$p=\frac{g}{3 h^{3}} \int p v f(p) \mathrm{d}^{3} p=\frac{g c^{2}}{3 h^{3}} \int \frac{p^{2}}{E} f(p) \mathrm{d}^{3} p$,

where we have used $v=p c^{2} / E$ [49]. The density of dark matter $\rho_{\chi}$ for the non-relativistic case with $E=m_{\chi} c^{2}$ and $P \approx m_{\chi} v_{\chi}$ is given by $\rho_{\chi}=m_{\chi} n_{\chi}$ whereas the dark matter pressure is given by

$p_{\chi}=\frac{g c^{2}}{3 h^{3}} \int \frac{p^{2}}{E} f(p) \mathrm{d}^{3} p \approx \frac{4 \pi g}{3 h^{3}} \int \frac{p^{4}}{m_{\chi}} \mathrm{d} p$, 
which leads to

$p_{\chi}=c^{2} \sigma^{2} \rho_{\chi}$,

where we have used $\sigma^{2}=<\vec{v}_{x}^{2}>/ 3 c^{2}, \sigma$ being the onedimensional velocity dispersion. By means of the dark matter conservation equation

$\dot{\rho}_{\chi}+3 \frac{\dot{a}}{a} \rho_{\chi}\left(1+\sigma^{2}\right)=0$,

we can find the following general solution:

$\rho_{\chi}=\frac{\rho_{\chi}, 0}{\left(a / a_{0}\right)^{3\left(1+\sigma^{2}\right)}}$,

where $\rho_{\chi, 0}$ is the density of dark matter at the present value of scale factor $a=a_{0}$. In the standard model, for the description of the dynamics of our universe with a normal dark matter we work with the following equation:

$\frac{\dot{a}^{2}}{a^{2}}=H_{0}^{2}\left[\frac{\Omega_{b, 0}}{\left(a / a_{0}\right)^{3}}+\frac{\Omega_{\mathrm{rad}, 0}}{\left(a / a_{0}\right)^{4}}+\frac{\Omega_{\chi, 0}}{\left(a / a_{0}\right)^{3\left(1+\sigma^{2}\right)}}+\Omega_{\Lambda}\right]$.

However, in the LQC model we will have the following modification:

$$
\begin{aligned}
\frac{\dot{a}^{2}}{a^{2}}= & H_{0}^{2}\left(\frac{\Omega_{b, 0}}{\left(a / a_{0}\right)^{3}}+\frac{\Omega_{\mathrm{rad}, 0}}{\left(a / a_{0}\right)^{4}}+\frac{\Omega_{\chi, 0}}{\left(a / a_{0}\right)^{3\left(1+\sigma^{2}\right)}}+\Omega_{\Lambda}\right) \\
& -\frac{H_{0}^{2}}{\rho_{c}}\left(\frac{\Omega_{b, 0}}{\left(a / a_{0}\right)^{3}}+\frac{\Omega_{\mathrm{rad}, 0}}{\left(a / a_{0}\right)^{4}}+\frac{\Omega_{\chi, 0}}{\left(a / a_{0}\right)^{3\left(1+\sigma^{2}\right)}}+\Omega_{\Lambda}\right)^{2} .
\end{aligned}
$$

Since we aim to extract numerical results for dynamical BEC quantities, we have to mention the values of several parameters and quantities such as the Hubble constant $H_{0}=70 \mathrm{~km} / \mathrm{s} / \mathrm{Mpc}=2.27 \times 10^{-18} \mathrm{~s}^{-1}$, the Hubble time $t_{H}=H_{0}^{-1}=4.39 \times 10^{17} \mathrm{~s}$, the critical density $\rho_{\mathrm{cr}, 0}=$ $9.24 \times 10^{-30} \mathrm{gr} / \mathrm{cm}^{3}$, and the present day dark matter densities $\Omega_{b, 0}=0.045, \Omega_{\chi, 0} \approx 0.228, \Omega_{\mathrm{rad}, 0}=8.24 \times 10^{-5}$, $\Omega_{\Lambda}=0.73$, respectively [50]. It is worth to recall that the following dark matter case is non-relativistic, so the global cosmological evolution of the universe will not be affected by the variation of the numerical values of $\sigma^{2}$.

As is explained in the introduction, at very low temperature all dilute Bose gas particles are condensed to the same quantum ground state which leads to the BEC formation. When the particle's wavelengths overlap, they will experience correlation with each other and the mean inter-particles distance $l$ becomes definitely smaller than the thermal wavelength $\lambda_{T}$. This is possible when the temperature is almost $T_{\text {cr }} \approx 2 \pi \hbar^{2} \rho^{2 / 3} / m^{5 / 3} k_{B}$, where $m$ is the particle mass in the condensate, $k_{\mathrm{B}}$ is Boltzmann's constant [51] and $\rho$ is the density. Under the condition that the universe is sufficiently large and the temperature is low enough, there will be a coherent state in progress due to the overlap of the particles wavelengths. Our main assumption is that the dark matter halos may be constructed by a strongly coupled dilute BEC at absolute zero. As a result, all the particles are in the condensate. Therefore in this situation, just binary collisions at low energy are considerable. These collisions are specified by just one parameter $l_{a}, s$-wave scattering length, which is thoroughly independent of the two-body potential. Consequently, the interaction potential can be replaced by an effective interaction, $V_{l}\left(\vec{r}^{\prime}-\vec{r}\right)=\lambda \delta\left(\vec{r}^{\prime}-\vec{r}\right)$, where $\lambda=4 \pi \hbar^{2} l_{a} / m_{\chi}$ [51]. The Gross-Pitaevskii (GP) equation, describing the ground state properties of the dark matter, for the dark matter halos can be obtained from the GP energy functional as follows:

$$
\begin{aligned}
E[\Psi]= & \int\left[\frac{\hbar^{2}}{2 m_{\chi}}|\nabla \Psi(\vec{r})|^{2}+\frac{U_{0}}{2}|\Psi(\vec{r})|^{4}\right] \mathrm{d} \vec{r} \\
& -\frac{1}{2} G m_{\chi}^{2} \iint \frac{|\Psi(\vec{r})|^{2}\left|\Psi\left(\vec{r}^{\prime}\right)\right|^{2}}{\left|\vec{r}-\vec{r}^{\prime}\right|} \mathrm{d} \vec{r} \mathrm{~d} \vec{r}^{\prime},
\end{aligned}
$$

where $U_{0}=4 \pi \hbar^{2} l_{a} / m_{\chi}[51]$ and obviously we have applied $\Psi(\vec{r})$ as the wave function of the condensate. In Eq. (22), the first and second terms are the quantum pressure and the interaction energy, respectively, and the third term is the gravitational potential energy. It should be noted that the condensate dark matter mass density is

$\rho_{\chi}(\vec{r})=m_{\chi}|\Psi(\vec{r})|^{2}=m_{\chi} \rho(\vec{r}, t)$

and that the wave function satisfies the normalization condition $N=\int|\Psi(\vec{r})|^{2} \mathrm{~d} \vec{r}$, where $N$ is the total number of particles. Eventually, by imposing the variational procedure $\delta E[\Psi]-\mu \delta \int|\Psi(\vec{r})|^{2} \mathrm{~d} \vec{r}=0$, the GP equation is extracted,

$$
\begin{aligned}
& -\frac{\hbar^{2}}{2 m_{\chi}} \nabla^{2} \Psi(\vec{r})+m_{\chi} V(\vec{r}) \Psi(\vec{r})+U_{0}|\Psi(\vec{r})|^{2} \Psi(\vec{r}) \\
& \quad=\mu \Psi(\vec{r}),
\end{aligned}
$$

while $\mu$ plays the role of the chemical potential. In addition the gravitational potential $V$ follows the well-known Poisson equation, $\nabla^{2} V=4 \pi G \rho$. Having considered the time dependency, the generalized GP equation, showing a gravitationally trapped rotating $\mathrm{BEC}$, is found as

$$
\begin{aligned}
& i \hbar \frac{\partial}{\partial t} \Psi(\vec{r}, t) \\
& \quad=\left[-\frac{\hbar^{2}}{2 m_{\chi}} \nabla^{2}+m_{\chi} V(\vec{r})+U_{0}|\Psi(\vec{r}, t)|^{2}\right] \Psi(\vec{r}, t) .
\end{aligned}
$$

By means of the well-known Madelung representation of the wave function, we can reach our purpose more easily than using the last generalized GP equation. In order to go through this, we should consider the following representation of $\Psi$ :

$\Psi(\vec{r}, t)=\sqrt{\rho(\vec{r}, t)} \exp \left[\frac{i}{\hbar} S(\vec{r}, t)\right]$, 
where $S(\vec{r}, t)$ represents the classical action. Inserting the last expression of $\Psi(\vec{r}, t)$ into Eq. (25) leads to the following differential equations:

$$
\begin{aligned}
\frac{\partial \rho_{\chi}}{\partial t}+\nabla \cdot\left(\rho_{\chi} \vec{v}\right)= & , \\
\rho_{\chi}\left[\frac{\partial \vec{v}}{\partial t}+(\vec{v} \cdot \nabla) \vec{v}\right]= & -\nabla p_{\chi}\left(\frac{\rho_{\chi}}{m_{\chi}}\right)-\rho_{\chi} \nabla\left(\frac{V}{m_{\chi}}\right) \\
& -\nabla V_{Q},
\end{aligned}
$$

where $\vec{v}=\nabla S / m_{\chi}$ is the velocity of the quantum fluid and $V_{Q}$ is the quantum potential, given by

$V_{Q}=-\left(\frac{\hbar^{2}}{2 m_{\chi}}\right) \frac{\nabla^{2} \sqrt{\rho \chi}}{\sqrt{\rho \chi}}$.

By defining $u_{0}=\frac{2 \pi \hbar^{2} l_{a}}{m_{\chi}^{3}}$, the condensate effective pressure is obtained

$p_{\chi}\left(\frac{\rho_{\chi}}{m_{\chi}}\right)=u_{0} \rho_{\chi}^{2}$.

The quantum pressure term makes a great contribution just close to the boundary of the condensate provided that the number of particles in the gravitationally bounded BEC becomes large enough. As a result, the quantum stress term in the condensate equation of motion can be ignored, which is the so-called Thomas-Fermi approximation. When the number of particles in the condensate goes to infinity, the mentioned Thomas-Fermi approximation becomes accurate. It is also related to the classical limit of the theory. According to its definition, the velocity field is irrotational, following the condition $\nabla \times \vec{v}=0$. In accordance with Eqs. (10) and (30) we can write

$\dot{\rho_{\chi}}+3 \rho_{\chi} \frac{\dot{a}}{a}\left(1+\frac{u_{0}}{c^{2}} \rho_{\chi}\right)=0$,

which has the following general solution with the integration constant $C_{\chi}$ :

$\rho_{\chi}=\frac{C_{\chi}}{\left(a / a_{0}\right)^{3}-\left(u_{0} / c^{2}\right) C_{\chi}}$

Having imposed the condition $\rho_{\chi}=\rho_{\chi, 0}$ for $a=a_{0}$, we can find the density of the condensate as

$\rho_{\chi}=\frac{c^{2}}{u_{0}} \frac{\rho_{0 \chi}}{\left(a / a_{0}\right)^{3}-\rho_{0 \chi}}$,

where

$\rho_{0 \chi}=\frac{\rho_{\chi, 0} u_{0} c^{2}}{1+\rho_{\chi, 0} u_{0} / c^{2}}$.

\section{Loop quantum cosmological dynamics of the Bose-Einstein condensation}

For an interacting Bose system case, where all dark matter particles are supposed to be in a single-particle state, the transition procedure from the normal to the condensed phase, and the order of the phase transition has been extensively studied in the recent literature $[33,52,53]$. In principle, BEC represents a spontaneous breaking of $U(1)$ gauge symmetry, with the condensate fraction showing the order parameter, leading to the second-order phase transition [54,55]. Nevertheless, it has been shown that BEC is a first-order phase transition $[56,57]$. From another point of view, in thermodynamics the first-order phase transitions result in a genuine mathematical singularity. The question "can finite systems in nature explicitly show such a behavior?" is a mysterious controversial question in physics [58]. Nonetheless, a significant study about the thermodynamic instability in an ideal Bose gas, confined in a cubic box, has shown that a system including a finite number of particles can exhibit an interrupted phase transition, describing a real mathematical singularity, with constant pressure [57]. Of course the mentioned result was obtained without considering a continuous approximation or a thermodynamic limit. Therefore, according to above discussion, we will assume that the intrinsic dynamics of BEC is described in the framework of a first-order phase transition.

\subsection{Cosmological parameters at the condensation point}

As we know, in general, the chemical potential $\mu$ in a physical system is a function which depends on the temperature and particle density $n=N / V$ ( $n$ is the total particle number and $V$ is the volume). By considering the property of extensivity of the Helmholtz free energy $F=F(N, V, T)$, we are allowed to write not only $F=V f(n, T)$ but also $F=$ $N \tilde{f}(v, T)$, where $v=V / N=n^{-1}$ and $f=n \tilde{f}$. Using the Helmholtz free energy we can obtain $\mu(n, T)=(\partial f / \partial n)_{T}$ and $p(n, T)=-(\partial \tilde{f} / \partial v)_{T}$, having the same information.

Imposing the thermodynamical requirements, the chemical potential and the pressure are supposed to be singlevalued which means that for arbitrary values of $n, v$, and $T$, there must be just one choice for $\mu$ or $p$, respectively [56]. Hence, the pressure at the transition moment during the BEC process must be continuous. Imposing this thermodynamic condition on Eqs. (18) and (30) helps us to fix the transition density $\rho_{\chi}^{\text {cr }}$ as follows:

$\rho_{\chi}^{\mathrm{cr}}=\frac{c^{2} \sigma^{2}}{u_{0}}=\frac{c^{2} \sigma^{2} m_{\chi}^{3}}{2 \pi \hbar^{2} l_{a}}$.

Apparently, in order to have the numerical value of the transition density, we should know the scattering length, the dark matter particle mass and the particle velocity disper- 
sion. Supposing typically the mass of dark matter particle, the mean velocity square, and the scattering length to be of the order of $1 \mathrm{eV}, 10^{15} \mathrm{~cm}^{2} / \mathrm{s}^{2}$, and $10^{-10} \mathrm{~cm}$, respectively, we can write

$$
\begin{aligned}
\rho_{\chi}^{\mathrm{cr}}= & 3.868 \times 10^{-21} \times\left(\frac{\sigma^{2}}{3 \times 10^{-6}}\right) \times\left(\frac{m_{\chi}}{10^{-33} \mathrm{~g}}\right)^{3} \\
& \times\left(\frac{l_{a}}{10^{-10} \mathrm{~cm}}\right)^{-1} \mathrm{~g} / \mathrm{cm}^{3} .
\end{aligned}
$$

Therefore, the critical temperature at the BEC moment is obtained by

$$
\begin{aligned}
T_{\mathrm{cr}} & \approx \frac{2 \pi \hbar^{2}}{\zeta(3 / 2)^{2 / 3} m_{\chi}^{5 / 3} k_{\mathrm{B}}}\left(\rho_{\chi}^{\mathrm{cr}}\right)^{2 / 3} \\
& =\frac{\left(2 \pi \hbar^{2}\right)^{1 / 3} c^{4 / 3}}{\zeta(3 / 2)^{2 / 3} k_{\mathrm{B}}} \frac{\left(\sigma^{2}\right)^{2 / 3} m_{\chi}^{1 / 3}}{l_{a}^{2 / 3}}
\end{aligned}
$$

where $\zeta(3 / 2)$ is the Riemann zeta function, or equivalently

$$
\begin{aligned}
T_{\mathrm{cr}} \approx & 6.57 \times 10^{3} \times\left(\frac{m_{\chi}}{10^{-33} \mathrm{~g}}\right)^{1 / 3} \times\left(\frac{\sigma^{2}}{3 \times 10^{-6}}\right)^{2 / 3} \\
& \times\left(\frac{l_{a}}{10^{-10} \mathrm{~cm}}\right)^{-2 / 3} K .
\end{aligned}
$$

The critical pressure of the dark matter fluid at the critical point is given by

$$
\begin{aligned}
p_{\mathrm{cr}}= & 1.04 \times 10^{-5} \times\left(\frac{\sigma^{2}}{3 \times 10^{-6}}\right)^{2} \times\left(\frac{m_{\chi}}{10^{-33} \mathrm{~g}}\right)^{3} \\
& \times\left(\frac{l_{a}}{10^{-10} \mathrm{~cm}}\right)^{-1} \text { dyne } / \mathrm{cm}^{2} .
\end{aligned}
$$

Using Eq. (35) we can calculate the critical value of the scale factor as

$$
\frac{a_{\mathrm{cr}}}{a_{0}}=\left(\frac{\rho_{\chi}, 0 u_{0}}{c^{2} \sigma^{2}}\right)^{1 / 3\left(1+\sigma^{2}\right)}=\left(\frac{2 \pi \hbar^{2} l_{a} \rho_{\mathrm{cr}, 0} \Omega_{\chi, 0}}{c^{2} \sigma^{2} m_{\chi}^{3}}\right)^{1 / 3\left(1+\sigma^{2}\right)}
$$

Consequently, we can find the critical redshift as follows:

$1+z_{\mathrm{cr}}=\left(\frac{2 \pi \hbar^{2} l_{a} \rho_{\mathrm{cr}, 0} \Omega_{\chi, 0}}{c^{2} \sigma^{2} m_{\chi}^{3}}\right)^{-1 / 3\left(1+\sigma^{2}\right)}$.

By inserting the above mentioned numerical values of the parameters in the two last relations we have

$$
\begin{aligned}
\frac{a_{\mathrm{cr}}}{a_{0}}= & 8.17 \times 10^{-4} \times\left(\frac{m_{\chi}}{10^{-33} g}\right)^{-\left(1+\sigma^{2}\right)} \\
& \times\left(\frac{\sigma^{2}}{3 \times 10^{-6}}\right)^{-1 / 3\left(1+\sigma^{2}\right)} \times\left(\frac{l_{a}}{10^{-10} \mathrm{~cm}}\right)^{1 / 3\left(1+\sigma^{2}\right)},
\end{aligned}
$$

and

$$
\begin{aligned}
1+z_{\mathrm{cr}}= & 1.22 \times 10^{3} \times\left(\frac{m_{\chi}}{10^{-33} g}\right)^{\left(1+\sigma^{2}\right)} \\
& \times\left(\frac{\sigma^{2}}{3 \times 10^{-6}}\right)
\end{aligned}
$$

The obtained critical values of density, temperature, pressure, scale factor, and redshift are important in the study of the subsequent LQC evolution of the universe during the BoseEinstein condensation toward the end time of the phase transition when the universe enters in the complete Bose-Einstein condensed dark matter phase. We will use these critical values in the study of this evolution in the following subsection.

\subsection{Loop quantum cosmological evolution during the Bose-Einstein condensation phase}

In the first-order phase transition, we have constant temperature and pressure, respectively as $T=T_{\mathrm{cr}}$ and $p=$ $p_{\text {cr. }}$. Moreover, the enthalpy $W=(\rho+p) a^{3}$ and entropy $S=s a^{3}$ are also conserved quantities. The phase transition begins through a decrease in the density of dark matter and increase in the density of Bose-Einstein condensed state. This is because the density $\rho_{\chi}^{\mathrm{cr}}\left(T_{\mathrm{cr}}\right) \equiv \rho_{\chi}^{\text {nor }}$ is converted to $\rho_{\chi}\left(T_{\mathrm{cr}}\right) \equiv \rho_{\chi}^{\mathrm{BEC}}$ during the phase transition. One may define a useful time dependent parameter $h(t)$, playing the role of $\rho_{\chi}(t)$, which is called the volume fraction of the matter in the Bose-Einstein condensed phase

$h(t)=\frac{\rho_{\chi}(t)-\rho_{\chi}^{\text {nor }}}{\rho_{\chi}^{\text {BEC }}-\rho_{\chi}^{\text {nor }}}$.

By simplifying (44), we can extract the time evolving dark matter energy density during the transition process as

$\rho_{\chi}(t)=\rho_{\chi}^{\mathrm{BEC}} h(t)+\rho_{\chi}^{\text {nor }}[1-h(t)]=\rho_{\chi}^{\text {nor }}\left[1+n_{\chi} h(t)\right]$,

where we have defined

$n_{\chi}=\frac{\rho_{\chi}^{\mathrm{BEC}}-\rho_{\chi}^{\text {nor }}}{\rho_{\chi}^{\text {nor }}}$

By looking at Eq. (44), we can determine the beginning and final points as $h\left(t_{\mathrm{cr}}\right)=0\left(t_{\mathrm{cr}}\right.$ being the beginning time of transition in which $\left.\rho_{\chi}\left(t_{\mathrm{cr}}\right) \equiv \rho_{\chi}^{\text {nor }}\right)$ and $h\left(t_{\mathrm{BEC}}\right)=1\left(t_{\mathrm{BEC}}\right.$ being the end time of transition in which $\left.\rho_{\chi}\left(t_{\mathrm{BEC}}\right) \equiv \rho_{\chi}^{\mathrm{BEC}}\right)$, respectively. At the end time of phase transition the universe enters in the Bose-Einstein condensed dark matter phase. As mentioned, by using $h(t)$ instead of $\rho_{\chi}(t)$ in Eq. (10), we can find the following relation: 
$\frac{\dot{a}}{a}=-\frac{1}{3} \frac{\left(\rho_{\chi}^{\mathrm{BEC}}-\rho_{\chi}^{\mathrm{nor}}\right) \dot{h}}{\rho_{\chi}^{\mathrm{nor}}+p_{\mathrm{cr}}+\left(\rho_{\chi}^{\mathrm{BEC}}-\rho_{\chi}^{\text {nor }}\right)}=-\frac{1}{3} \frac{r \dot{h}}{1+r h}$,

where

$r=\frac{\rho_{\chi}^{\mathrm{BEC}}-\rho_{\chi}^{\text {nor }}}{\rho_{\chi}^{\text {nor }}+p_{\mathrm{cr}}}=\frac{n_{\chi}}{1+p_{\mathrm{cr}} / \rho_{\chi}^{\text {nor }}}$.

The last expression implies that in general we have $r<0$, $r \in(-1,0)$, and also $n_{\chi}<0$, because $\rho_{\chi}^{\mathrm{BEC}}<\rho_{\chi}^{\text {nor }}$. Clearly, imposing the condition $h\left(t_{\mathrm{cr}}\right)=0$, we can obtain the relation between the scale factor and $h(t)$ from Eq. (47) as

$a(t)=a_{\mathrm{cr}}[1+r h(t)]^{-1 / 3}, \quad t \in\left(t_{\mathrm{cr}}, t_{\mathrm{BEC}}\right)$,

where $a_{\mathrm{cr}}=a\left(t_{\mathrm{cr}}\right)$. The value of the scale factor at the end of phase transition is as follows:

$a_{\mathrm{BEC}}=a\left(t_{\mathrm{BEC}}\right)=a_{\mathrm{cr}}(1+r)^{-1 / 3}$.
This is an important result, since this condensation process has modified the expansion rate of the universe. Subsequently, we can find the evolution equations for the baryonic matter and the radiation density during the phase transition, respectively, as

$\rho_{b}=\frac{\rho_{b, 0}}{\left(a_{\mathrm{cr}} / a_{0}\right)^{3}}[1+r h(t)], t \in\left(t_{\mathrm{cr}, \mathrm{t}_{\mathrm{BEC}}}\right)$

and

$\rho_{\mathrm{rad}}=\frac{\rho_{\mathrm{rad}, 0}}{\left(a_{\mathrm{cr}} / a_{0}\right)^{4}}[1+r h(t)]^{\frac{4}{3}}, t \in\left(t_{\mathrm{cr}, \mathrm{t}_{\mathrm{BEC}}}\right)$.

Using all these equations, we consider the time evolution of the volume fraction of the condensed matter dark energy density during the BEC process, which describes the dynamics of this process in the cosmic history in the LQC model,

$$
\begin{aligned}
\frac{\mathrm{d} h}{\mathrm{~d} \tau}= & -3\left(\frac{1}{r}+h\right) \sqrt{\frac{\Omega_{b, 0}}{\left(\frac{a_{\mathrm{cr}}}{a_{0}}\right)^{3}}(1+r h)+\frac{\Omega_{\mathrm{rad}, 0}}{\left(\frac{a_{\mathrm{cr}}}{a_{0}}\right)^{4}}(1+r h)^{4 / 3}+\Omega_{\chi, \text { nor }}\left(1+n_{\chi} h\right)+\Omega_{\Lambda}} \\
& \times \sqrt{1-\frac{1}{\rho_{c}}\left(\frac{\Omega_{b, 0}}{\left(\frac{a_{\mathrm{cr}}}{a_{0}}\right)^{3}}(1+r h)+\frac{\Omega_{\mathrm{rad}, 0}}{\left(\frac{a_{\mathrm{cr}}}{a_{0}}\right)^{4}}(1+r h)^{4 / 3}+\Omega_{\chi, \text { nor }}\left(1+n_{\chi} h\right)+\Omega_{\Lambda}\right)} .
\end{aligned}
$$

Here, we have used $\tau$ as a dimensionless time variable $\tau=H_{0} t$ and $\Omega_{\chi, \text { nor }}=\rho_{\chi}^{\text {nor }} / \rho_{\mathrm{cr}, 0}$. Considering Eq. (48) and $p_{\mathrm{cr}} / \rho_{\chi}^{\text {nor }}=\sigma^{2} \ll 1$, we deduce the approximation $r \approx n_{\chi}$ in the following calculations. Another approximation comes from neglecting the radiation energy density contribution, so Eq. (53) can be integrated to extract $h(t)$

$$
\begin{aligned}
h(t)= & \frac{\Omega_{\Lambda}^{2}}{r \Omega_{\mathrm{tr}}}\left(\frac{\rho_{c}^{2}}{4 \Omega_{\Lambda}^{4}}\left(\Omega_{\mathrm{tr}}+\Omega_{\Lambda}\right)\left(\Omega_{\mathrm{tr}}+\Omega_{\Lambda}-\rho_{c}\right) \frac{(-1+M)^{2}}{4 \Omega_{\Lambda}^{4}\left(\rho_{c}-\Omega_{\Lambda}\right)}\left(\Omega_{\mathrm{tr}} \rho_{c}-2\left(\Omega_{\mathrm{tr}}-\rho_{c}\right) \Omega_{\Lambda}-2 \Omega_{\Lambda}^{2}\right)^{2}\right. \\
& \left.+\frac{(-1+M) M}{\Omega_{\Lambda}^{4} \sqrt{\Omega_{\Lambda}\left(1-\frac{\Omega_{\Lambda}}{\rho_{c}}\right)}} \sqrt{-\left(\Omega_{\Lambda}+\Omega_{\mathrm{tr}}\right)\left(\Omega_{\Lambda}+\Omega_{\mathrm{tr}}-\rho_{c}\right)\left(\Omega_{\mathrm{tr}} \rho_{c}-2\left(\Omega_{\mathrm{tr}}-\rho_{c}\right) \Omega_{\Lambda}-2 \Omega_{\Lambda}^{2}\right)}\right)^{\frac{1}{2}} \\
& -\frac{\Omega_{\Lambda}+\Omega_{\mathrm{tr}}}{r \Omega_{\mathrm{tr}}}+\frac{\rho_{c}}{2 r \Omega_{\mathrm{tr}}}
\end{aligned}
$$


where we have defined

$\Omega_{\mathrm{tr}}=\frac{\Omega_{b, 0}}{\left(\frac{a_{\mathrm{cr}}}{a_{0}}\right)^{3}}+\Omega_{\chi, \mathrm{nor}}$,

and

$M=e^{3 H_{0}\left(t-t_{c r}\right) \sqrt{\Omega_{\Lambda}\left(1-\frac{\Omega_{\Lambda}}{\rho_{c}}\right)}}$.

In order to obtain the necessary time interval for the entire converting process of the total normal dark matter into the Bose-Einstein condensed phase, we put $h\left(t=t_{\text {BEC }}\right)=1$ in our last calculation,

According to (33), we can obtain the cosmological density of the dark matter as follows:

$\rho_{\chi}^{\mathrm{BEC}}=\frac{c^{2}}{u_{0}} \frac{\rho_{0 \chi}(1+r)}{\left(a_{\mathrm{cr}} / a_{0}\right)^{3}-\rho_{0 \chi}(1+r)}$,

where the numerical value of $\rho_{0 \chi}$ is obtained from (34),

$$
\rho_{0 \chi}=\frac{1.63 \times 10^{-15} \times\left(l_{a} / 10^{-10} \mathrm{~cm}\right)\left(\mathrm{m} / 10^{-33} \mathrm{gr}\right)^{-3}}{1+1.63 \times 10^{-15} \times\left(l_{a} / 10^{-10} \mathrm{~cm}\right)\left(\mathrm{m} / 10^{-33} \mathrm{gr}\right)^{-3}} .
$$

$$
\begin{aligned}
\Delta t_{\mathrm{con}}= & t_{\mathrm{BEC}}-t_{\mathrm{cr}}=\frac{t_{H}}{3 \Omega_{\Lambda}} \times \sqrt{\frac{\Omega_{\Lambda}}{1-\frac{\Omega_{\Lambda}}{\rho_{c}}}} \\
& \times \ln \left[\frac{2 \sqrt{\Omega_{\Lambda}\left(1-\frac{\Omega_{\Lambda}}{\rho_{c}}\right)} \sqrt{\left(\frac{-\Omega_{\mathrm{tr}}^{2}}{\rho_{c}}+\Omega_{\mathrm{tr}}-2 \frac{\Omega_{\mathrm{tr}} \Omega_{\Lambda}}{\rho_{c}}\right)(1+r)+\Omega_{\Lambda}\left(1-\frac{\Omega_{\Lambda}}{\rho_{c}}\right)+2 \Omega_{\Lambda}\left(1-\frac{\Omega_{\Lambda} \Omega_{\mathrm{tr}}}{\rho_{c}}\right)+\Omega_{\mathrm{tr}}}}{(1+r)\left(2 \sqrt{\Omega_{\Lambda}\left(1-\frac{\Omega_{\Lambda}}{\rho_{c}}\right)} \sqrt{\frac{-\Omega_{\mathrm{tr}}^{2}}{\rho_{c}}+\Omega_{\mathrm{tr}}-2 \frac{\Omega_{\mathrm{tr}} \Omega_{\Lambda}}{\rho_{c}}+\Omega_{\Lambda}\left(1-\frac{\Omega_{\Lambda}}{\rho_{c}}\right)+2 \Omega_{\Lambda}\left(1-\frac{\Omega_{\Lambda}+\Omega_{\mathrm{tr}}}{\rho_{c}}\right)+\Omega_{\mathrm{tr}}}\right)}\right] .
\end{aligned}
$$

By inserting the value of dark energy density parameter $\Omega_{\Lambda}$ as 0.68 and also considering the standard values $\rho_{c} \simeq$ $0.82 M_{p}^{4} \simeq 1.84 \times 10^{170} \mathrm{eV} / \mathrm{cm}^{3}, \ell_{a}=10^{-10} \mathrm{~cm}, m=1$ $\mathrm{eV}$, and $\Omega_{\mathrm{tr}}=5.02 \times 10^{8}$, we can obtain the following expression for the conversion time of the dark matter to the Bose-Einstein condensed phase:

$$
\begin{aligned}
\Delta t_{\text {cond }}= & 0.39 \\
& \times \ln \left[\frac{0.99+1.135 \times 10^{-9} \sqrt{5.02 \times 10^{8}+5.02 \times 10^{8} r}}{1+r}\right] \times t_{H} \mathrm{~s} .
\end{aligned}
$$

For instance, for $r=-0.2$ and $t_{H}=4.39 \times 10^{17} \mathrm{~s}$, we will have $\Delta t_{\text {cond }} \simeq 3.82 \times 10^{16}$ s. Since it is thoroughly useful to depict the time evolution of $h(t)$, we have represented (54) for different values of $r$ in Fig. 1 .

\subsection{The post-condensation phase in LQC model}

After the complete conversion to the BEC phase, the postcondensation phase begins, which starts at $t=t_{\mathrm{BEC}}$ and $a_{\mathrm{BEC}}=a_{\mathrm{cr}}(1+r)^{-1 / 3}$. Similar to the era, during the condensation phase, in which the cosmological dynamics is affected by the BEC (see (50)), the Bose-Einstein condensed dark matter, in the post-condensation phase, may change the cosmological dynamics of the universe in LQC model. Moreover, the Bose-Einstein condensed dark matter may have considerable impact on the subsequent structure formation in the universe. Therefore, the post-condensation phase and the corresponding cosmological values in LQC model are important and deserve more scrutiny.
Clearly, the density (59) should be positive, which leads to the condition $\left(a_{\mathrm{cr}} / a_{0}\right)>_{\rho 0 \chi^{1 / 3}}(1+r)^{1 / 3}$. Simplifying Eq. (21) and defining $\Omega_{\mathrm{BE}}=\frac{\Omega_{\chi, 0}}{1+\rho_{\chi}, 0 u_{0} / c^{2}}$ gives us the time evolution of the scale factor in the BEC phase as follows:

$$
\begin{aligned}
\frac{\mathrm{d}\left(a / a_{0}\right)}{\mathrm{d} t}= & H_{0} \sqrt{\Omega_{B E}} \frac{a / a_{0}}{\sqrt{\left(a / a_{0}\right)^{3}-\rho_{0 \chi}}} \\
& \times \sqrt{1-\frac{H_{0}^{2}}{\rho_{c}} \frac{\Omega_{B E}}{\left(a / a_{0}\right)^{3}-\rho_{0 \chi}}} .
\end{aligned}
$$

By integration of this relation, we obtain exactly an expression of the following form:

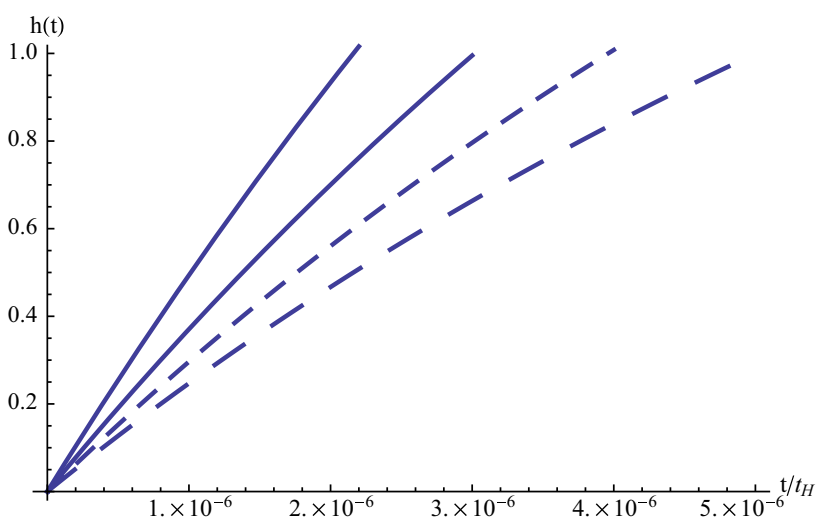

Fig. 1 Time evolution of the condensed dark matter fraction $h(t)$ for different values of $r: r=-0.15$ (solid curve), $r=-0.20$ (dotted curve), $r=-0.25$ (dashed curve), and $r=-0.30$ (long dashed curve), respectively 


$$
\begin{aligned}
& \sqrt{\Omega_{\mathrm{BE}}} H_{0}(t-C)=\frac{2}{3} \sqrt{\left(a / a_{0}\right)^{3}-\rho_{0 \chi}} \sqrt{\frac{-H_{0}^{2} \Omega_{\mathrm{BE}}+\left(a / a_{0}\right)^{3} \rho_{c}-\rho_{0 \chi} \rho_{c}}{\rho_{c}\left(\left(a / a_{0}\right)^{3}-\rho_{0 \chi}\right)}} \\
& -\frac{2 \rho_{0 \chi} \rho_{c} \sqrt{\left(a / a_{0}\right)^{3}-\rho_{0 \chi}} \sqrt{\frac{\rho_{c}\left(\left(a / a_{0}\right)^{3}-\rho_{0 \chi}\right)-H_{0}^{2} \Omega_{\mathrm{BE}}}{\rho_{c}\left(\left(a / a_{0}\right)^{3}-\rho_{0 \chi}\right)}} \tanh ^{-1}\left(\sqrt{1-\frac{\left(a / a_{0}\right)^{3} \rho_{c}}{H_{0}^{2} \Omega_{\mathrm{BE}}+\rho_{0 \chi} \rho_{c}}}\right)}{3\left(H_{0}^{2} \Omega_{\mathrm{BE}}+\rho_{0 \chi} \rho_{c}\right) \sqrt{1-\frac{\left(a / a_{0}\right)^{3} \rho_{c}}{H_{0}^{2} \Omega_{\mathrm{BE}}+\rho_{0 \chi} \rho_{c}}}}
\end{aligned}
$$

where $C$ is an integration constant, determined from the con-

straint $a=a_{\mathrm{BEC}}$ at $t=t_{\mathrm{BEC}}$ as

$$
\begin{gathered}
C=t_{\mathrm{BEC}}-\frac{2}{3 H_{0} \sqrt{\Omega_{B E}}} \sqrt{\left(a_{\mathrm{BEC}} / a_{0}\right)^{3}-\rho_{0 \chi}} \sqrt{\frac{-H_{0}^{2} \Omega_{\mathrm{BE}}+\left(a_{\mathrm{BEC}} / a_{0}\right)^{3} \rho_{c}-\rho_{0 \chi} \rho_{c}}{\rho_{c}\left(\left(a_{\mathrm{BEC}} / a_{0}\right)^{3}-\rho_{0 \chi}\right)}} \\
-\frac{2 \rho_{0 \chi} \rho_{c} \sqrt{\left(a_{\mathrm{BEC}} / a_{0}\right)^{3}-\rho_{0 \chi}} \sqrt{\frac{\rho_{c}\left(\left(a_{\mathrm{BEC}} / a_{0}\right)^{3}-\rho_{0 \chi}\right)-H_{0}^{2} \Omega_{\mathrm{BE}}}{\rho_{c}\left(\left(a_{\mathrm{BEC}} / a_{0}\right)^{3}-\rho_{0 \chi}\right)}} \tanh ^{-1}\left(\sqrt{1-\frac{\left(a_{\mathrm{BEC}} / a_{0}\right)^{3} \rho_{c}}{H_{0}^{2} \Omega_{\mathrm{BE}}+\rho_{0 \chi} \rho_{c}}}\right)}{3 H_{0} \sqrt{\Omega_{B E}}\left(H_{0}^{2} \Omega_{\mathrm{BE}}+\rho_{0 \chi} \rho_{c}\right) \sqrt{1-\frac{\left(a_{\mathrm{BEC}} / a_{0}\right)^{3} \rho_{c}}{H_{0}^{2} \Omega_{\mathrm{BE}}+\rho_{0 \chi} \rho_{c}}}}
\end{gathered}
$$

For the universe presented here, filled with radiation, dark energy, baryonic matter, and Bose-Einstein condensed dark matter, the equation describing time evolution of the scale factor is given by

$$
\begin{aligned}
& \frac{1}{\left(a / a_{0}\right)} \frac{\mathrm{d}\left(a / a_{0}\right)}{\mathrm{d} t} \\
& =H_{0} \sqrt{\frac{\Omega_{b, 0}}{\left(\frac{a}{a_{0}}\right)^{3}}+\frac{\Omega_{\mathrm{rad}, 0}}{\left(\frac{a}{a_{0}}\right)^{4}}+\frac{\Omega_{\mathrm{BE}}}{\left(\frac{a}{a_{0}}\right)^{3}-\rho_{0, \chi}}+\Omega_{\Lambda}} \\
& \quad \times \sqrt{1-\frac{H_{0}^{2}}{\sqrt{\rho_{c}}}\left(\frac{\Omega_{b, 0}}{\left(a / a_{0}\right)^{3}}+\frac{\Omega_{\mathrm{rad}, 0}}{\left(a / a_{0}\right)^{4}}+\frac{\Omega_{\mathrm{BE}}}{\left(a / a_{0}\right)^{3}-\rho_{0 \chi}}+\Omega_{\Lambda}\right)}, \\
& t \geq t_{\mathrm{BEC},}
\end{aligned}
$$

which can be integrated by considering the initial conditions $a\left(t_{\mathrm{BEC}}\right)=a(0)=a_{\mathrm{BEC}}$. To study the time evolution of the scale factor in such a universe which contains BEC dark matter, it will be useful to depict it for different values of the $\rho_{0 \chi}$ in Fig. 2. It is remarkable that the existence of the condensed dark matter changes the cosmological dynamics of the universe in the post-condensation phase, and the magnitude of this change decreases with the increase of the Bose-Einstein condensate parameter $\rho_{0 x}$. This behavior is expected because as $\rho_{0 \chi}$ increases, the corresponding gravity contributes stronger to the dynamics and decreases the expansion rate. On the other hand, it is seen from Fig. 2 that as $\rho_{0 \chi}$ increases, the Bose-Einstein condensation phase (where $a=a_{\mathrm{BEC}}$ ) occurs at earlier times. This is also justified because as $\rho_{0 \chi}$ increases, the $\rho_{\chi}^{\mathrm{BEC}}$ increases as well and so the scale factor $a_{\mathrm{BEC}}$ corresponding to this increased density tends toward the scale factors having higher density, which naturally occurs at earlier times.

\section{Conclusions}

When the Bose-Einstein condensation happens, dark matter contains two phases, the normal and the condensed phase, respectively. Under a thermodynamic condition, we expect to have the continuity of the pressure profile of these two phases right at the beginning of the condensation process.

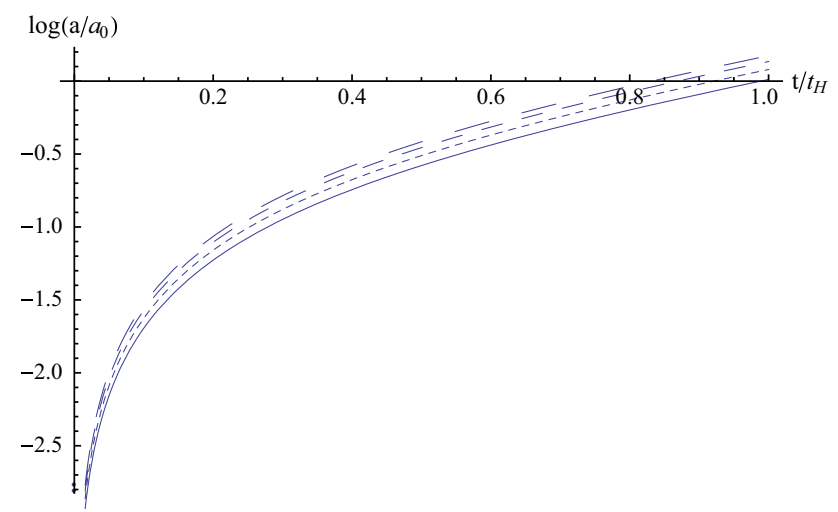

Fig. 2 Time evolution (in a logarithmic scale) of the scale factor of the universe in the post-Bose-Einstein condensation phase, for $a_{\mathrm{BEC}}=$ $a(0)=8.1 \times 10^{-4}(z \approx 1200)$, and for different values of $\rho_{0 \chi}=10^{-11}$ $\mathrm{eV} / \mathrm{cm}^{3}$ (solid curve), $\rho_{0 \chi}=5 \times 10^{-11} \mathrm{eV} / \mathrm{cm}^{3}$ (dotted curve), $\rho_{0 \chi}=$ $10^{-10} \mathrm{eV} / \mathrm{cm}^{3}$ (dashed curve), and $\rho_{0 \chi}=5 \times 10^{-10} \mathrm{eV} / \mathrm{cm}^{3}$ (long dashed curve), respectively 
This leads to uniquely determination of dark matter density at the condensation time as well as other thermodynamical quantities, like temperature and pressure. The explicit numerical values of the condensation quantities is related to mean square velocity of the normal dark matter $\sigma^{2}$ and the mass $m_{\chi}$ of the dark matter particle and the scattering length $l_{a}$. As a result of the uncertainty of the values of $\sigma^{2}$, $m_{\chi}$, and $l_{a}$, apparently it is difficult to predict the precise and exact cosmological moment of the Bose-Einstein condensation and consequently the corresponding cosmological quantities. Nevertheless, by considering some "standard" numerical values, it will be possible to obtain a total qualitative picture of the transition. Consequently, we estimate the values of the dark matter mass and the mean velocity of the non-relativistic dark matter particles, of the order of $1 \mathrm{eV}$ [19,59] and $900 \mathrm{~km} / \mathrm{s}$, respectively. According to the general analysis of the condensation process, the first phase of condensation belongs to the standard $\Lambda$ cold dark matter $(\Lambda \mathrm{CDM})$ model. In this paper, we have generalized this analysis and have gone through the loop quantum cosmology context. With the standard numerical values of the dark matter quantities, the condensation was begun at a redshift around $z=1200$. Here, we have studied the time evolution of the fraction factor $h(t)$ of the condensed dark matter through the loop quantum cosmology. In the next step, we have extracted the modified phase transition time interval in LQC, which is approximately of the order of Hubble time and shows the viability of our results. We have studied the evolution during the Bose-Einstein condensation phase and found that this condensation process modifies the expansion rate of the universe. Also, we have studied the post-condensation phase in LQC model, describing the time evolution of the universe right after the end of phase transition, which leads to (64). The cosmic time dependence of the global formation of BEC dark matter (at $t_{\mathrm{BEC}}$ ) is an advantage, compared to the ordinary dark matter, which makes BEC dark matter to be preferred than the ordinary dark matter. This is an important feature which makes BEC dark matter to be similar to the global dark matter candidates like neutrinos, WIMPs, axions and LSP. The ordinary dark matter has not such capability and cannot form global sources of dark matter at a definite cosmic time, and that is why we have favored BEC dark matter in the present study. We have plotted the time behavior of Eqs. (53) and (64) in Figs. 1 and 2 for different values of $r$ and $\rho_{0 \chi}$, showing the definite effect of the phase transition and Bose-Einstein condensed dark matter density in the modified cosmological history in the loop quantum cosmology.

A major point regarding the present study is that assuming two phases of dark matter, one as the bosonic particles before BEC, and the other one as BEC dark matter, one may ask: "is there any experimental indication for the occurrence of $\mathrm{BEC}$, the existence of these two phases, and the preference of one to the other one?" and "why is it necessary to distinguish ordinary dark matter from BEC dark matter?". Of course, at present there is no direct evidence for the occurrence of BEC, the existence of these two phases, and the preference of one to the other one. However, in order to answer properly these questions, we may try to answer the alternative question: "is there any indication for distinguishing between the dynamics of the universe having bosonic particles without the occurrence of BEC, and the one with BEC dark matter". In this regard, we may point out that the time evolution of the scale factor with BEC is different from that without BEC for many possible reasons, such as: (1) the equation of state parameter of BEC dark matter is different from that of bosonic particles without BEC, due to the first-order phase transition, (2) in the absence of BEC, the bosonic particles may interact or participate in some possible transformations into other matter components, like visible matter or dark energy, each resulting in different dynamical history of the universe; however, with BEC the condensed bosonic particles cannot decay into other forms of matter and they remain as the BEC dark matter permanently, with a definite dynamical history distinguishable from the variety of dynamics corresponding to the regular bosonic particles having different kinds of interaction with other matter components.

Acknowledgments This work has been supported financially by Research Institute for Astronomy and Astrophysics of Maragha (RIAAM) under research Project No. 1/4165-96.

Open Access This article is distributed under the terms of the Creative Commons Attribution 4.0 International License (http://creativecomm ons.org/licenses/by/4.0/), which permits unrestricted use, distribution, and reproduction in any medium, provided you give appropriate credit to the original author(s) and the source, provide a link to the Creative Commons license, and indicate if changes were made. Funded by SCOAP ${ }^{3}$.

\section{References}

1. K. Bamba, J. de Haro, S.D. Odintsov, JCAP 1302, 008 (2013)

2. A. Ashtekar, J. Lewandowski, J. Math. Phys. 36, 2170 (1995)

3. A. Ashtekar, J. Lewandowski, Class. Quant. Grav. 21, R53 (2004)

4. C. Rovelli, Liv. Rev. Rel. 1, 1 (1998)

5. A. Ashtekar et al., Adv. Theor. Math. Phys. 7, 233 (2003)

6. P. Wu, S.N. Zhang, JCAP 0806, 007 (2008)

7. X. Fu, H. Yu, P. Wu, Phys. Rev. D 78, 063001 (2008)

8. S. Chen, B. Wang, J. Jing, Phys. Rev. D 78, 123503 (2008)

9. R. Lamon, A. Woehr, Phys. Rev. D 81, 024026 (2010)

10. S. Li, Y. Ma, Eur. Phys. J. C 68, 227 (2010)

11. M. Jamil, D. Momeni, M.A. Rashid, Eur. Phys. J. C 71, 1711 (2011)

12. M. Jamil, U. Debnath, Astrophys. Space. Sci 333, 3 (2011)

13. J. de Haro, Y.-F. Cai, Gen. Rel. Grav 47, 8 (2015)

14. J. de Haro, J. Amorós, JCAP 08, 025 (2014)

15. J. de Haro, A.N. Makarenko, A.N. Myagky, S.D. Odintsov, V.K. Oikonomou, Phys. Rev. D 92, 124026 (2015)

16. J.J. Amorós, J. de Haro, S.D. Odintsov, Phys. Rev. D 89, 104010 (2014)

17. T. Fukuyama, M. Morikawa, Prog. Theor. Phys. 115, 1047 (2006) 
18. T. Fukuyama, M. Morikawa, T. Tatekawa, J. Cosmol. Astropart. Phys. 06, 033 (2008)

19. T. Fukuyama, M. Morikawa, Phys. Rev. D 80, 063520 (2009)

20. E.A. Cornell, C.E. Wieman, Rev. Mod. Phys. 74, 875 (2002)

21. W. Ketterle, Rev. Mod. Phys. 74, 1131 (2002)

22. L. Pitaevskii, S. Stringari, Bose-Einstein Condensation (Clarendon Press, Oxford, 2003)

23. R.A. Duine, H.T.C. Stoof, Phys. Rep. 396, 115 (2014)

24. Q. Chen, J. Stajic, S. Tan, K. Levin, Phys. Rep. 412, 1 (2005)

25. C.J. Pethick, H. Smith, Bose-Einstein Condensation in Dilute Gases (Cambridge University Press, Cambridge, 2008)

26. M.Y. Khlopov, B.A. Malomed, Y.B. Zeldovich, Mon. Not. Roy. Astr. Soc 215, 575 (1985)

27. Z.G. Berezhiani, M.Y. Khlopov, Z. Phys. C 49, 73 (1991)

28. Z.G. Berezhiani, A.S. Sakharov, M.Y. Khlopov, Yadernaya Fizika 55, 1918 (1992). [English translation: Sov. J. Nucl. Phys, 55, 1063 (1992)]

29. A.S. Sakharov, D.D. Sokoloff, M.Y. Khlopov, Yadernaya Fizika 59, 1050 (1996). [English translation: Phys. Atom. Nucl, 59, 1005 (1996)]

30. M.Y. Khlopov, A.S. Sakharov, D.D. Sokoloff, Nucl. Phys. B (Proc. Suppl.) 72, 105 (1999)

31. I.G. Dymnikova, M.Y. Khlopov, Mod. Phys. Lett. A 15, 2305 (2000)

32. I.G. Dymnikova, M.Y. Khlopov, Eur. Phys. J. C 20, 139 (2001)

33. T. Harko, Phys. Rev. D 83, 123515 (2011)

34. A. Ashtekar, D. Sloan, Gen. Rel. Grav. 43, 3619 (2011)

35. M. Bojowald, H.H. Hernández, M. Kagan, P. Singh, A. Skirzewski, Phys. Rev. D 74, 123512 (2006)

36. M. Bojowald, Living Rev. Rel. 8, 11 (2005)

37. M. Bojowald, J. Phys. Conf. Ser. 24, 77 (2005)

38. M. Bojowald, H.A. Morales-Tecotl, Lect. Notes Phys. 646, 421 (2004)
39. M. Bojowald, A. Skirzewski, Rev. Math. Phys. 18, 713 (2006)

40. K.A. Meissner, Class. Quant. Grav. 21, 5245 (2004)

41. A. Ghosh, P. Mitra, Phys. Rev. D 71, 027502 (2005)

42. A. Ashtekar, T. Pawlowski, P. Singh, Phys. Rev. Lett. 96, 141301 (2006)

43. A. Ashtekar, T. Pawlowski, P. Singh, Phys. Rev. D 74, 084003 (2006)

44. A. Ashtekar, T. Pawlowski, P. Singh, K. Vandersloot, Phys. Rev. D 75, 024035 (2007)

45. K. Vandersloot, Phys. Rev. D 75, 023523 (2007)

46. A. Corichi, T. Vukasinac, J.A. Zapata, AIP Conf. Proc. 977, 64-71 (2008)

47. C.J. Hogan, J.J. Dalcanton, Phys. Rev. D 62, 063511 (2000)

48. J. Madsen, Phys. Rev. D 64, 027301 (2001)

49. S.L. Shapiro, S.A. Teukolsky, Black holes, White Dwarfs, and Neutron Stars: The Physics of Compact Objects (Wiley, New York, 1983)

50. G. Hinshaw et al., Astrophys. J. Suppl. Ser. 180, 225 (2009)

51. F. Dalfovo, S. Giorgini, L.P. Pitaevskii, S. Stringar, Rev. Mod. Phys. 71, 463 (1999)

52. T. Harko, Mon. Not. R. Astron. Soc. 413, 3095 (2011)

53. P.-H. Chavanis. arXiv:1103.2698 [gr-qc]

54. J.O. Anderson, Rev. Mod. Phys. 76, 599 (2004)

55. E.H. Lieb, R. Seiringer, J. Yngvason, Phys. Rev. Lett. 94, 080401 (2005)

56. L. Olivares-Quiroz, V. Romero-Rochin, J. Phys. B 43, 205302 (2010)

57. J.-H. Park, S.-W. Kim, Phys. Rev. A 81, 063636 (2010)

58. M. Kastner, Rev. Mod. Phys. 80, 167 (2008)

59. D. Boyanovsky, H.J. de Vega, N.G. Sanchez, Phys. Rev. D 77, 043518 (2008) 